Pacific Journal of Mathematic 


\section{SOME ISOLATED SUBSETS OF INFINITE SOLVABLE GROUPS}

\section{S. PASSMAN}

The main theorem of this paper offers necessary and sufficient conditions for a solvable group $G$ to be covered by a finite union of certain types of isolated subsets. This result will have applications to the study of the semisimplicity problem for group rings of solvable groups.

Let $H$ be a subgroup of $G$. We define

$$
\sqrt[G]{H}=\sqrt{H}=\left\{x \in G \mid x^{m} \in H \text { for some } m \geqq 1\right\} \text {. }
$$

Observe that $\sqrt{H}$ need not be a subgroup of $G$ even if $G$ is solvable. We say that $H$ has locally finite index in $G$ and write $[G: H]=l . f$. if for every finitely generated subgroup $L$ of $G$ we have $[L: L \cap H]<\infty$. Suppose $[G: H]=l . f$. and let $x \in G$. Then $[\langle x\rangle:\langle x\rangle \cap H]<\infty$ so $x^{m} \in H$ for some $m \geqq 1$ and $x \in \sqrt{H}$. Thus $G=\sqrt{H}$. The main result of this paper is a generalized converse of this fact for solvable groups $G$.

THEOREM. Let $G$ be a solvable group and let $H_{1}, H_{2}, \cdots, H_{n}$ be subgroups with

$$
G=\bigcup_{1}^{n} \sqrt{H_{i}} .
$$

Then for some $i=1,2, \cdots, n$ we have $\left[G: H_{i}\right]=l . f$.

This paper constitutes one third of the solution of the semisimplicity problem for group rings of solvable groups. The remaining two thirds can be found in [1] and [4]. Moreover a description of this latter result as well as an analogue of the above theorem for linear groups will appear in [3].

We first list some basic properties of subgroups of locally finite index.

LEMmA 1. Let $G \supseteqq W \supseteqq H, G \supseteqq W_{1} \supseteqq H_{1}$ and let $N \triangleleft G$.

(i) $[G: H]=l . f$. implies $[G: W]=l . f$.

(ii) $[G / N: H N / N]=l . f$. implies $[G: H N]=l . f$.

(iii) $[W: H]=l . f$. implies $[W N: H N]=l . f$.

(iv) $[W: H]=l . f$. and $\left[W_{1}: H_{1}\right]=l . f$. implies $\left[W \cap W_{1}: H \cap H_{1}\right]=l . f$.

(v) $[G: W]=l . f$. and $[W: H]=l . f$. implies $[G: H]=l . f$.

Proof. (i) If $L \leqq G$ then [ $L: W \cap L] \leqq[L: H \cap L]$ so this is clear. (ii) Let $L$ be a finitely generated subgroup of $G$. Then $L N / N$ 
is finitely generated so

$$
[L N / N:(H N / N) \cap(L N / N)]<\infty .
$$

Thus $[L N: H N \cap L N]<\infty$. Since $L \subseteq L N$ this yields $[L: H N \cap L]<\infty$.

(iii) Let $L$ be a finitely generated subgroup of $W N$. Then there exists a finitely generated subgroup $S \subseteq W$ with $L N=S N$. Now $[S: S \cap H]<\infty$ so $[S N:(S \cap H) N]<\infty$. Observe that $(S \cap H) N \subseteq$ $S N \cap H N$ so $[S N: S N \cap H N]<\infty$. Finally $L \cong L N=S N$ yields $[L: L \cap H N]<\infty$ and [WN:HN] $=l . f$.

(iv) Let $L$ be a finitely generated subgroup of $W \cap W_{1}$. Then $L \subseteq W$ yields $[L: H \cap L]<\infty$ and similarly $\left[L: H_{1} \cap L\right]<\infty$. Thus $\left[L:\left(H \cap H_{1}\right) \cap L\right]<\infty$ and $\left[W \cap W_{1}: H \cap H_{1}\right]=l . f$.

(v) Finally let $L$ be a finitely generated subgroup of $G$. Since $[G: W]=l . f$. we have $[L: L \cap W]<\infty$. Thus by [1, Lemma 6.1] $L \cap W$ is finitely generated and since $[W: H]=l . f$. we have

$$
[L \cap W: L \cap W \cap H]<\infty \text {. }
$$

This yields $[L: L \cap H]<\infty$ and the lemma is proved.

Lemma 2. Let $A H$ be a group with $A$ a normal abelian subgroup. Set

$$
B=\left\{a \in A \mid\left[H: H \cap H^{a}\right]=l . f .\right\} .
$$

Then we have

(i) $A \cap H \triangleleft A H$

(ii) if $a \in A$ then $H \cap H^{a}=N_{H}(a(H \cap A))$

(iii) $B$ is a subgroup of $A$ and $B \triangleleft A H$.

(iv) if $[A: B]<\infty$ and $B /(A \cap H)$ is torsion, then $[A H: H]=l$.f.

Proof. (i) Since $A \triangleleft A H$ we have $A \cap H \triangleleft H$. Since $A$ is abelian we have $A \cap H \triangleleft A$. Thus $A \cap H \triangleleft A H$.

(ii) Let $h \in H \cap H^{a}$. Then $h \in H$ and $h^{a^{-1}} \in H$ so $h^{-1} h^{a^{-1}} \in H \cap A$ since $A$ is normal. Thus $h$ centralizes $a$ modulo $H \cap A$ so $h$ normalizes $a(H \cap A)$ and $H \cap H^{a} \leqq N_{H}(a(H \cap A))$.

Let $h \in N_{H}(a(H \cap A))$. Then $h \in H$ and $h^{a} \equiv h$ modulo $H \cap A$. Since $H \cap A \triangleleft A H$ we have $H^{a} \supseteq H \cap A$ and $h \in H^{a}(H \cap A)=H^{a}$. Thus $h \in H \cap H^{a}$.

(iii) Clearly $1 \in B$. Since $[\alpha(H \cap A)]^{-1}=a^{-1}(H \cap A)$ we see that $N_{H}(a(H \cap A))=N_{H}\left(a^{-1}(H \cap A)\right)$. Thus $a \in B$ implies $a^{-1} \in B$. Finally let $a, b \in B$. Then $\left[H: H \cap H^{a}\right]=$ l.f. implies $\left[H^{b}: H^{b} \cap H^{a b}\right]=$ l.f. so by Lemma 1 (iv), $\left[H \cap H^{b}: H \cap H^{b} \cap H^{a b}\right]=l . f$. Now $\left[H: H \cap H^{b}\right]=$ l.f. so Lemma $1(v)$ yields $\left[H: H \cap H^{b} \cap H^{a b}\right]=l$.f. Since $H \cap H^{a b} \supseteqq$ $H \cap H^{b} \cap H^{a b}$ we have $\left[H: H \cap H^{a b}\right]=l . f$. and $B$ is a group. Clearly $B \triangleleft A H$. 
(iv) By Lemma 1 (ii) since $A \cap H \triangleleft A H, A \cap H \subseteq B, A \cap H \subseteq H$ it clearly suffices to work in $A H /(A \cap H)$ or in other words we may assume that $A \cap H=\langle 1\rangle$. Thus $A H$ is the semidirect product of $A$ by $H$. Now [AH:BH] $<\infty$ so by Lemma 1 (v) it suffices to show that $[B H: H]=$ l.f.

Let $L$ be a finitely generated subgroup of $B H$. Then there exists a finitely generated subgroup $B_{1}$ of $B$ and a finitely generated subgroup $H_{1}$ of $H$ such that $L \subseteq B_{1}^{H_{1}} \cdot H_{1}$. By definition of $B$ and by (ii) each element of $B_{1}$ has only finitely many conjugates under the action of $H_{1}$. Thus $B_{1}^{H_{1}}$ is a finitely generated abelian group. Since this group is torsion by assumption we have

$$
\left|B_{1}^{H_{1}}\right|<\infty \text { and }\left[B_{1}^{H_{1}} \cdot H_{1}: H_{1}\right]=\left|B_{1}^{H_{1}}\right|<\infty \text {. }
$$

Finally $L \cong B_{1}^{H_{1}} \cdot H_{1}$ so $\left[L: L \cap H_{1}\right]<\infty$. Since $L \cap H=L \cap\left(B_{1}^{H_{1}} \cdot H_{1}\right) \cap$ $H=L \cap H_{1}$, the result follows.

We can now obtain the main result.

Proof of the Theorem. By induction on $d(G)$, the derived length of $G$. If $d(G)=0$ then $G=\langle 1\rangle$ so the result is clear. Assume the result for all groups $G$ with $d(G) \leqq d$. For any group $G$ let $D G=$ $G^{(d)}$ be the $d$ th derived subgroup of $G$.

Suppose $d(G)=d+1$. Since $G=\bigcup_{1}^{n} \sqrt{H_{i}}$ we have clearly

$$
G /(D G)=\bigcup_{1}^{n} \sqrt{H_{i}(D G) /(D G)} \text {. }
$$

By induction some of these groups have locally finite index in $G /(D G)$. Thus by Lemma 1 (ii) we have for a suitable ordering of the $H_{i}$ 's that $\left[G: H_{i}(D G)\right]=l . f$. for $i=1,2, \cdots, s$ (some $s \geqq 1$ ) and $\left[G: H_{i}(D G)\right] \neq$ l.f. for $i>s$. We call $s$ the parameter of the situation and we prove the $d(G)=d+1$ case by induction on the parameter starting with $s=0$ which does not occur.

Assume the result for all groups $G$ with either $d(G) \leqq d$ or $d(G)=$ $d+1$ and parameter $<s$. Now fix $G$ and suppose $d(G)=d+1, G=$ $\mathrm{U}_{1}^{n} \sqrt{H_{i}}$ and the parameter of this situation is $s$. Set $A=D G$ so $A$ is a normal abelian subgroup of $G$ and say $H_{1} A, H_{2} A, \cdots, H_{s} A$ have locally finite index in $G$. For each $i \leqq s$ set

$$
B_{i}=\left\{a \in A \mid\left[H_{i}: H_{i} \cap H_{i}^{a}\right]=l . f .\right\} .
$$

By Lemma 2 (iii) $B_{i}$ is a subgroup of $A$.

Step 1. For each $i \leqq s$ set

$$
A_{1 i}=\left\{a \in A \mid\left[H_{1}: H_{i}^{a} \cap H_{1}\right]=l_{. f} \cdot\right\} \text {. }
$$


Then $A=\bigcup_{1}^{s} A_{1 i}$.

Proof. Fix $a \in A$ and let $x \in H_{1}$. Then $\left(a x a^{-1}\right)^{m} \in H_{j}$ for some $j$ so $x^{m} \in H_{j}^{a} \cap H_{1}$. Thus

$$
H_{1}=\bigcup_{1}^{n} \sqrt[H_{1}]{H_{i}^{a} \cap H_{1}} .
$$

If $d\left(H_{1}\right) \leqq d$ then by induction $\left[H_{1}: H_{i}^{a} \cap H_{1}\right]=l . f$. for some $i$ and as in the argument below $i \leqq s$ so $a \in A_{1 i}$. Assume that $d\left(H_{1}\right)=d+1$ and consider the parameter of this situation. Observe that $D H_{1} \subseteq A \cap H_{1}$.

Suppose $\left[H_{1}:\left(H_{1}^{a} \cap H_{1}\right) D H_{1}\right]=$ l.f. Now $H_{1} \supseteq D H_{1}$ and $H_{1}^{a} \supseteqq\left(D H_{1}\right)^{a}=$ $D H_{1}$ since $A$ is abelian. Thus $\left(H_{1}^{a} \cap H_{1}\right) D H_{1}=H_{1}^{a} \cap H_{1}$ so $\left[H_{1}: H_{1}^{a} \cap\right.$ $\left.H_{1}\right]=l . f$. and $a \in A_{11}$.

Thus we may suppose that $\left[H_{1}:\left(H_{1}^{a} \cap H_{1}\right) D H_{1}\right] \neq l$.f. Let $\left[H_{1}\right.$ : $\left.\left(H_{j}^{a} \cap H_{1}\right) D H_{1}\right]=l$.f. Since $A$ is normal in $G$ and $A \supseteqq D H_{1}$ we have by Lemma 1 (iii)

$$
\left[H_{1} A:\left(H_{j}^{a} \cap H_{1}\right) A\right]=\left[H_{1} A:\left(H_{j}^{a} \cap H_{1}\right)\left(D H_{1}\right) A\right]=\text { l.f. }
$$

Now $\left[G: H_{1} A\right]=l . f$. so by Lemma $1(\mathrm{v})$ we have $\left[G:\left(H_{j}^{a} \cap H_{1}\right) A\right]=l . f$. Now $H_{j} A \supseteqq\left(H_{1} \cap H_{j}^{a}\right) A$ so $\left[G: H_{j} A\right]=$ l.f. by Lemma 1 (i) and $j \leqq s$. Since $j \neq 1$ the parameter of this situation is $<s$.

By induction $\left[H_{1}: H_{1} \cap H_{i}^{a}\right]=l . f$. for some $i \leqq n$. But then by Lemma 1 (i) $\left[H_{1}:\left(H_{1} \cap H_{i}^{a}\right) D H_{1}\right]=l$.f. so $i \leqq s$ by the above. Thus $a \in A_{1 i}$.

Step 2. If $A_{1 i} \neq \varnothing$ and $a_{i} \in A_{1 i}$ then $A_{1 i}=B_{i} a_{i}$.

Proof. Suppose $A_{1 i} \neq \varnothing$ and fix $a_{i} \in A_{1 i}$ and let $a \in A_{1 i}$. Then [ $\left.H_{1}: H_{i}^{a} \cap H_{1}\right]=$ l.f. and $\left[H_{1}: H_{i}^{a_{i}} \cap H_{1}\right]=$ l.f. yield by Lemma 1 (iii) (iv) first $\left[H_{1}: H_{1} \cap H_{i}^{a} \cap H_{i}^{a_{i}}\right]=$ l.f. and then $\left[H_{1} A:\left(H_{1} \cap H_{i}^{a} \cap H_{i}^{a}\right) A\right]=$ l.f. Since $\left[G: H_{1} A\right]=$ l.f. we have by Lemma 1 (v) $\left[G:\left(H_{1} \cap H_{i}^{a} \cap\right.\right.$ $\left.\left.H_{i}^{a}\right) A\right]=l . f$. Now

$$
\left(H_{1} \cap H_{i}^{a} \cap H_{i}^{a}\right) A \subseteq\left(H_{i}^{a} \cap H_{i}^{a}\right) A=\left(H_{i} \cap H_{i}^{a a} \bar{i}^{-1}\right) A
$$

so we have by Lemma 1 (i) (iv) $\left[G:\left(H_{i} \cap H_{i}^{a a{ }^{-1}}\right) A\right]=l . f$. and

$$
\left[H_{i}: H_{i} \cap\left(H_{i} \cap H_{i}^{a a}{ }^{-1}\right) A\right]=\text { l.f. }
$$

Observe that $H_{i} \cap H_{i}^{a{ }^{a}} \bar{i}^{-1} \supseteqq H_{i} \cap A$ and thus

$$
H_{i} \cap\left(H_{i} \cap H_{i}^{a a_{\bar{i}}^{-1}}\right) A=\left(H_{i} \cap H_{i}^{a{ }^{-1}}\right)\left(H_{i} \cap A\right)=H_{i} \cap H_{i}^{\alpha a-\bar{i}^{-1}} .
$$

Therefore the above yields $\left[H_{i}: H_{i} \cap H_{i}^{a a_{i}^{-1}}\right]=l . f$. so $a a_{i}^{-1} \in B_{i}$ and $a \epsilon$ $B_{i} a_{i}$. Hence $A_{1 i} \subseteq B_{i} a_{i}$.

Now let $b \in B_{i}$. Then $\left[H_{i}: H_{i}^{b} \cap H_{i}\right]=$ l.f. yields $\left[H_{i}^{a}{ }^{a}: H_{i}^{b a_{i}} \cap H_{i}^{a_{i}}\right]=$ 
l.f. so by Lemma 1 (iv) $\left[H_{1} \cap H_{i}^{a_{i}}: H_{1} \cap H_{i}^{b a_{i}} \cap H_{i}^{a_{i}}\right]=l . f$. Since [ $\left.H_{1}: H_{1} \cap H_{i}^{a_{i}}\right]=$ l.f. Lemma 1 (v) yields $\left[H_{1}: H_{1} \cap H_{i}^{b a_{i}} \cap H_{i}^{a_{i}}\right]=$ l.f. Since $H_{1} \cap H_{i}^{b a_{i}} \supseteqq H_{1} \cap H_{i}^{b a_{i}} \cap H_{i}^{a_{i}}$ we have $\left[H_{1}: H_{1} \cap H_{i}^{b a_{i}}\right]=l . f$. and $b a_{i} \in A_{1 i}$. Thus $B_{i} a_{i} \subseteq A_{1 i}$ and this fact follows.

Step 3. We may assume that for all $i=1,2, \cdots, s$ we have [A: $\left.B_{i}\right]<\infty$ and $B_{i} /\left(A \cap H_{i}\right)$ not torsion.

Proof. By Steps 1 and 2 we have

$$
A=\cup B_{i} a_{i} \text { over all } A_{1 i} \neq \varnothing
$$

and hence by Lemma 5.2 of [1]

$$
A=\bigcup B_{i} a_{i} \text { over all } A_{1 i} \neq \varnothing, \quad\left[A: B_{i}\right]<\infty .
$$

In particular since $1 \in A$ there exists $k \leqq s$ with $\left[A: B_{k}\right]<\infty$ and $1 \in A_{1 k}$.

Suppose $k \neq 1$. Then $1 \in A_{1 k}$ implies that $\left[H_{1}: H_{k} \cap H_{1}\right]=l_{. f}$. and hence as we observed earlier this yields $\sqrt[H_{1}]{H_{k} \cap H_{1}}=H_{1}$. Since this clearly yields $\sqrt[G]{H_{1}} \subseteq \sqrt[G]{H_{k}}$ we then have $G=\bigcup_{2}^{n} \sqrt{H_{i}}$. Observe that here $\left[G: H_{i}(D G)\right]=l . f$. precisely for $i=2,3, \cdots, s$ so that parameter of this new situation is $s-1$. By induction $\left[G: H_{i}\right]=l . f$. for some $i$ and the result follows. Thus we may assume that $k=1$. Hence [A: $\left.B_{1}\right]<\infty$.

Note that $B_{1} \supseteqq A \cap H_{1}$ since $A \cap H_{1} \triangleleft A H_{1}$. If $B_{1} /\left(A \cap H_{1}\right)$ is torsion then Lemma 2 (iv) implies that $\left[H_{1} A: H_{1}\right]=$ l.f. Since $\left[G: H_{1} A\right]=$ l.f. we conclude by Lemma $1(\mathrm{v})$ that $\left[G: H_{1}\right]=l . f$. and the result follows again. Thus we may assume that $B_{1} /\left(A \cap H_{1}\right)$ is not torsion.

In a similar manner for each $j \leqq s$ we can define sets $A_{j i}$ for $i=1,2, \cdots, s$ and conclude that we may assume $\left[A: B_{j}\right]<\infty$ and $B_{j} /\left(A \cap H_{j}\right)$ is not torsion.

Step 4. Completion of the proof.

Proof. Now $A$ is abelian so $\sqrt[A]{A \cap H_{i}}$ is a group. Since $A \neq$ $\sqrt[A]{A \cap H_{i}}$ for $i \leqq s$ by Step 3 we cannot even have $\left[A: \sqrt[A]{A \cap H_{i}}\right]<\infty$. Thus by Lemma 1.2 of [2], $A \neq \bigcup_{1}^{s} \sqrt[A]{A \cap H_{i}}$ so choose $a \in A, a \notin$ $\sqrt[4]{A \cap H_{i}}$ for all $i \leqq s$.

Let $B=B_{1} \cap B_{2} \cap \cdots \cap B_{s}$. Then $[A: B]<\infty$ and say $a^{t}=b \in B$ with $t \geqq 1$. Then clearly $b \notin \sqrt[A]{A \cap H_{i}}$ for all $i \leqq s$. For each $i \leqq s$ let $E_{i}=H_{i} \cap H_{i}^{b}=N_{H_{i}}\left(b\left(H_{i} \cap A\right)\right)$ by Lemma 2 (ii). Then $b \in B_{i}$ implies that $\left[H_{i}: E_{i}\right]=$ l.f. so by Lemma 1 (iii) (v) since $\left[G: H_{i} A\right]=$ l.f. we have $\left[G: E_{i} A\right]=$ l.f. Observe that $A$ abelian implies that 
$E_{i} A \subseteq N_{G}\left(b\left(H_{i} \cap A\right)\right)$. If $E=\bigcap_{1}^{s} E_{1} A$ then by Lemma 1 (iv), [G: $\left.E\right]=$ l.f.

Let $e \in E$. Now $G=\bigcup_{1}^{n} \sqrt{H_{i}}$ so for the $n+1$ elements $e, b e, b^{2} e$, $\cdots, b^{n} e$ there exists integers $m_{j}, k_{j} \geqq 1$ with

$$
\left(b^{j} e\right)^{m_{j}} \in H_{k_{j}} \text { for } j=0,1, \cdots, n \text {. }
$$

By the pigeon hole principle there exists $i \neq j$ with $\left(b^{i} e\right)^{m_{i}},\left(b^{j} e\right)^{m_{j}}$ both in $H_{k}$. Thus if $m=m_{i} m_{j}$ then $\left(b^{i} e\right)^{m},\left(b^{j} e\right)^{m}$ both belong to $H_{k}$.

Suppose that $k \leqq s$. Now $e \in E \subseteq E_{k} A \subseteq H_{k} A$ so $e$ normalizes the cosets $b\left(H_{k} \cap A\right)$ and $\left(H_{k} \cap A\right)$. Thus

$$
\left(b^{i} e\right)^{m} \in b^{i m} e^{m}\left(H_{k} \cap A\right), \quad\left(b^{i} e\right)^{m} \in H_{k}
$$

so $b^{i m} e^{m} \in H_{k}$. Similarly $b^{j m} e^{m} \in H_{k}$ and hence $b^{(i-j) m}=\left(b^{i m} e^{m}\right)\left(b^{j m} e^{m}\right)^{-1} \in$ $H_{k}$, a contradiction since $(i-j) m \neq 0$ and $b \notin \sqrt{H_{k} \cap A}$. Thus $k>s$.

Since $\left(b^{i} e\right)^{m} \in H_{k}$ for $k>s$ and $b \in A$ we see that $e^{m} \in H_{k} A$ and hence $E=\bigcup_{s+1}^{n} \sqrt[E]{H_{k} A \cap E}$. Thus $E / A=\bigcup_{s+1}^{n} \sqrt{\left(H_{k} A \cap E\right) / A}$. Since $D E \subseteq A$ we have $d(E / A) \leqq d$ so by induction and Lemma 1 (ii), $\left[E: H_{k} A \cap E\right]=l . f$. for some $k>s$. Since $[G: E]=l . f$. we then have by Lemma 1 (v) (i) $\left[G: H_{k} A\right]=l . f$. for some $k>s$. However this contradicts the definition of the parameter $s$ and the theorem is proved.

We close with a few comments about the theorem and proof.

First, some assumption on $G$ is obviously needed in the theorem. For example let $G$ be the finitely generated infinite $p$-group constructed by E. S. Golod (see Corollary 27.5 of [2]). Then $G=\sqrt{\langle 1\rangle}$ but $[G:\langle 1\rangle] \neq$ l.f.

Second, one might be tempted to guess that the appropriate definition of locally finite index should be $[G: H]=\widetilde{l . f}$. if and only if $[\langle H, S\rangle: H]<\infty$ for every finite subset $S$ of $G$. However this is not the right condition here. For example let $G=Z_{p}$ ? $Z_{p_{\infty}}$ and let $H=Z_{p \infty}$. Then $G$ is solvable and periodic so $G=\sqrt{H}$ but

$$
\left[\left\langle H, Z_{p}\right\rangle: H\right]=\infty \text {. }
$$

Third, it is interesting to observe in the proof that if $G \neq\langle 1\rangle$ is abelian, then $G=A$ so the results of the first three steps are trivial in this case. The proof for $G=A$ is contained in the first paragraph of the fourth step.

Finally, we remark that the proof of the special case of this result in which $G$ is assumed to equal $\sqrt{H}$ is very much simpler.

\section{REFERENCES}

1. C. R. Hampton and D. S. Passman, On the semisimplicity of group rings of solvable groups, Trans. Amer. Math. Soc., 173 (1972), 289-301. 
2. D. S. Passman, Infinite Group Rings, Marcel Dekker, New York, 1971.

3. D. S. Passman, On the semisimplicity of group rings of linear groups, Pacific. J. Math., to appear.

4. A. E. Zalesskii, A semisimplicity criteria for the group ring of a solvable group (in Russian), Doklady Akad. Nauk CCCP, to appear.

Received December 23, 1971. Research supported by N. S. F. Contract GP 29432.

UNIVERSITY OF WISCONSIN 



\section{PACIFIC JOURNAL OF MATHEMATICS}

EDITORS

\author{
H. SAMELSON \\ Stanford University \\ Stanford, California 94305 \\ C. R. HOBBY \\ University of Washington \\ Seattle, Washington 98105
}

\author{
J. DUGUNDJI \\ Department of Mathematics \\ University of Southern California \\ Los Angeles, California 90007 \\ RICHARD ARENS \\ University of California \\ Los Angeles, California 90024
}

\section{ASSOCIATE EDITORS}

E. F. BECKENBACH

B. H. NeUManN

F. WOLF

K. YosHIDA

\section{SUPPORTING INSTITUTIONS}

UNIVERSITY OF BRITISH COLUMBIA

CALIFORNIA INSTITUTE OF TECHNOLOGY

UNIVERSITY OF CALIFORNIA

MONTANA STATE UNIVERSITY

UNIVERSITY OF NEVADA

NEW MEXICO STATE UNIVERSITY

OREGON STATE UNIVERSITY

UNIVERSITY OF OREGON

OSAKA UNIVERSITY
UNIVERSITY OF SOUTHERN CALIFORNIA

STANFORD UNIVERSITY

UNIVERSITY OF TOKYO

UNIVERSITY OF UTAH

WASHINGTON STATE UNIVERSITY UNIVERSITY OF WASHINGTON

AMERICAN MATHEMATICAL SOCIETY NAVAL WEAPONS CENTER 


\section{Pacific Journal of Mathematics}

\section{Vol. 45, No. $1 \quad$ September, 1973}

William George Bade, Complementation problems for the Baire classes .......... 1

Ian Douglas Brown, Representation of finitely generated nilpotent groups ........ 13

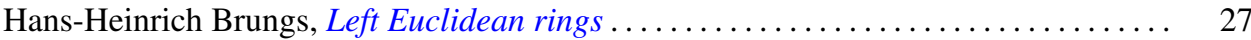

Victor P. Camillo and John Cozzens, A theorem on Noetherian hereditary rings ..... 35

James Cecil Cantrell, Codimension one embeddings of manifolds with locally flat

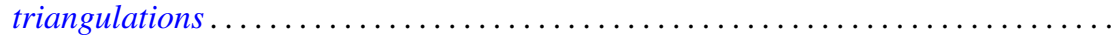

L. Carlitz, Enumeration of up-down permutations by number of rises . . . . . . . . . .

Thomas Ashland Chapman, Surgery and handle straightening in Hilbert cube

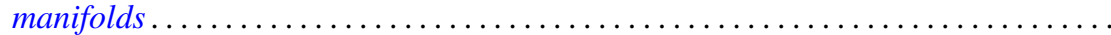

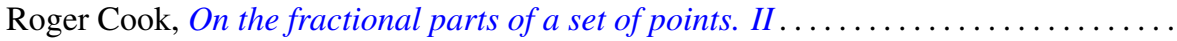

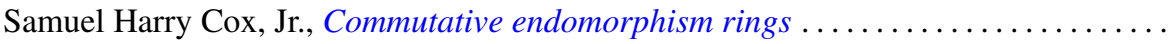

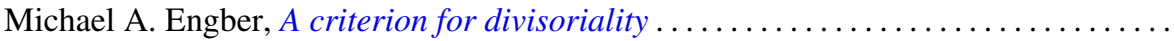

Carl Clifton Faith, When are proper cyclics injective . . . . . . . . . . . . . . 97

David Finkel, Local control and factorization of the focal subgroup . . . . . . . . . 113

Theodore William Gamelin and John Brady Garnett, Bounded approximation by

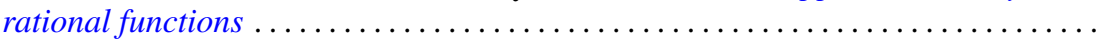

Kazimierz Goebel, On the minimal displacement of points under Lipschitzian

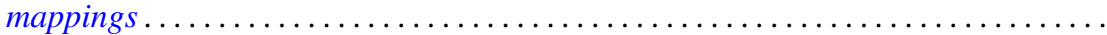

Frederick Paul Greenleaf and Martin Allen Moskowitz, Cyclic vectors for representations associated with positive definite measures: nonseparable

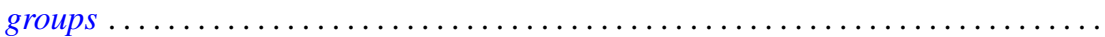

Thomas Guy Hallam and Nelson Onuchic, Asymptotic relations between perturbed linear systems of ordinary differential equations .

David Kent Harrison and Hoyt D. Warner, Infinite primes of fields and completions. .

James Michael Hornell, Divisorial complete intersections . ......

Jan W. Jaworowski, Equivariant extensions of maps ..............

John Jobe, Dendrites, dimension, and the inverse arc function .. .

Gerald William Johnson and David Lee Skoug, Feynman integrals of non-factorable

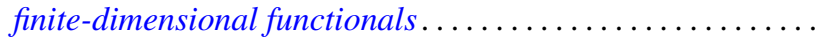

Dong S. Kim, A boundary for the algebras of bounded holomorphic functions ...... 269

Abel Klein, Renormalized products of the generalized free field and its derivatives ... 275

Joseph Michael Lambert, Simultaneous approximation and interpolation in $L_{1}$ and

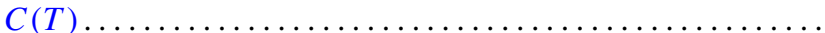

Kelly Denis McKennon, Multipliers of type $(p, p)$ and multipliers of the group

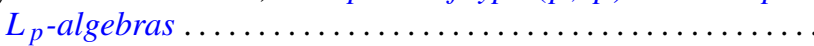

William Charles Nemitz and Thomas Paul Whaley, Varieties of implicative

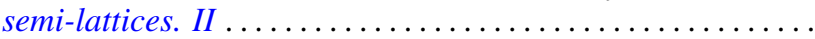

Donald Steven Passman, Some isolated subsets of infinite solvable

Norma Mary Piacun and Li Pi Su, Wallman compactifications on E-completely

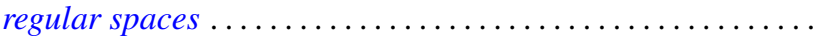

Jack Ray Porter and Charles I. Votaw, $S(\alpha)$ spaces and regular Hausdorff extensions....

Gary Sampson, Two-sided $L_{p}$ estimates of convolution transforms .

Ralph Edwin Showalter, Equations with operators forming a rig
Raymond Earl Smithson, Fixed points in partially ordered sets .

Victor Snaith and John James Ucci, Three remarks on symmetric products and

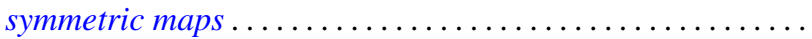

